\title{
Calculation of Nonlinear Stiffness of Rubber Pad under Different Temperatures and Prepressures
}

\author{
Chuanbo Xu, ${ }^{1,2}$ Mao-Ru Chi $\mathbb{D}^{1},{ }^{1}$ Liangcheng Dai $\mathbb{D},{ }^{1}$ and Zhaotuan Guo ${ }^{1}$ \\ ${ }^{1}$ State Key Laboratory of Traction Power, Southwest Jiaotong University, Chengdu, China \\ ${ }^{2}$ Henan Engineering Research Center of Rail Transit Intelligent Security, Zhengzhou Railway Vocational \& Technical College, \\ Zhengzhou, China \\ Correspondence should be addressed to Mao-Ru Chi; cmr2000@163.com
}

Received 23 October 2019; Revised 29 May 2020; Accepted 19 June 2020; Published 4 July 2020

Academic Editor: Nicola Nisticò

Copyright (c) 2020 Chuanbo Xu et al. This is an open access article distributed under the Creative Commons Attribution License, which permits unrestricted use, distribution, and reproduction in any medium, provided the original work is properly cited.

\begin{abstract}
The static stiffness of rubber springs is affected by temperature and prepressure. In this thesis, the relationship between Young's modulus and temperature of rubber was studied, and the quantitative relationship between them was determined. The approximate formula for calculating the static stiffness of rubber pads was further modified, and the ellipse approximation method and convexity coefficient correction method were proposed. In addition, the influence of temperature on geometric nonlinearity was considered. The formula for calculating nonlinear stiffness includes two variables: temperature and prepressure. The results of tests and theoretical calculations demonstrate that the nonlinear formula can be a good approximation and that it can meet the requirements of engineering applications.
\end{abstract}

\section{Introduction}

Rubber components are simple in structure and have buffering and damping effects; hence, they are widely used in vehicles, mechanical equipment, aerospace, and other fields. With the rapid development of high-speed railway, the requirements of running stability and comfort of high-speed trains have continuously increased. Therefore, it is necessary to accurately calculate the stiffness of rubber springs to effectively calculate and predict the performance of the trains. With the increasing geographical range covered by the trains, the operating temperature range of the rubber springs is large, normally ranging from $-60^{\circ} \mathrm{C}$ to $+60^{\circ} \mathrm{C}$. The operating conditions and carrying capacity of the trains are continuously changing. Consequently, the preload on the rubber spring is constantly changing. The change in static stiffness of rubber springs with temperature and prepressure should be expressed by an appropriate formula, which will be of great significance in further research on the calculation of dynamic performance.

The calculation of the mechanical properties of rubber springs has always been the focus of research, but previous studies have focused mainly on the dynamic stiffness and damping. Most models adopt different combinations of elements such as springs, dampers, friction, and fractional order, and the main influencing factors considered are frequency and amplitude [1-8]. Currently, the research on static stiffness of rubber springs, under the condition of small deformation at room temperature, is relatively mature. For rubber springs with different shapes, there are several theories and empirical formulas for calculation; most of these formulas adopt linear stiffness or consider a certain geometric nonlinear stiffness. Additionally, finite element method is widely used in the study of rubber constitutive model. This method can accurately simulate small static deformation experiments, which are calculated using strain energy and tensor function $[9,10]$, but the parameters calculated by computer simulation still depend on experimental data. Gajewski [11] presented finite element modeling theory for large deformation and analyzed the effectiveness of different models. Moreover, he [12] proposed a method to estimate the energy dissipation capacity of elastic materials under large deformation; these results are significant in the study of large deformations. Cardone and 
Gesualdi [13] studied the relationship between the properties of rubber elastomer and temperature, where they focused mainly on shear modulus, and the temperature was in the range of $20^{\circ} \mathrm{C}$ to $60^{\circ} \mathrm{C}$. However, there are few studies on low-temperature performance. In addition, there are large errors in theoretical calculations involving large deformations and few studies on temperature and prepressure as variables in the same time.

Stevenson [14, 15] studied the relationship between low-temperature crystallization and elastic modulus of natural rubber. The results showed that the elastic modulus of natural rubber increased significantly with low-temperature crystallization. In addition, the relationship between low-temperature deformation and crystallization was also studied. The authors of [16-18] analyzed and studied the performance of rubber springs at low temperature; the results showed that low temperature has a significant effect on the properties of rubber. Österlöf et al. [19-21] studied the relationship between the rubber model and temperature. Ding et al. [22] modified the low-temperature modulus of rubber and proposed a method for predicting low-temperature stiffness. Kari $[23,24]$ proposed a nonlinear temperature model of rubber pad based on shape coefficient and studied the influence of temperature on material geometric parameters at a certain prepressure. In the equation, approximation using an equivalent cylinder was used to calculate the shape coefficient. You et al. [25] studied the lowtemperature stiffness of laminated rubber, and the results showed that low temperature has a large influence on the vertical compression stiffness of laminated rubber; the horizontal compression stiffness is mainly affected by the quality and manufacturing process of rubber. Cheng et al. $[26,27]$ studied the nonlinear relationship between stiffness and prestress on different forms of rubber springs.

Accurate calculation of static stiffness is important for calculating the hyperelastic part of the dynamic performance of rubber. Therefore, in this thesis, the rubber pad is taken as an example to analyze the material nonlinearity and geometric nonlinearity; the formula for calculating vertical static stiffness, under the combined influence of temperature and prepressure, is established. The formula can predict the static stiffness of rubber more accurately, can meet the requirements of engineering applications, and is of great significance in the theoretical research of dynamic stiffness.

\section{Study of Relationship between Temperature and Young's Modulus}

2.1. Calculation of Young's Modulus Based on Statistical Theory. Rubber is a type of hyperelastic material; for an ideal rubber material, the conformational entropy of molecular net chain can be used for elastic analysis. The microscopic theory starts with the actual structure, the complex structure is simplified using some assumptions, and the ideal structure is constructed to facilitate calculation and analysis. Based on certain experimental results, the following assumptions are made:
(1) The intersection points are fixed at their average positions

(2) Microdeformations and macrodeformations are in the same proportion; thus, affine deformation is considered

(3) The chain of intersection points is a Gaussian chain, which conforms to the Gaussian statistical law

(4) The internal energy of the system is independent of the conformation of each chain

According to the statistical theory of elasticity of rubber,

$$
E=\frac{\sigma}{\varepsilon}=\frac{3 \rho R_{\mathrm{air}} T}{\bar{M}_{\mathrm{C}}},
$$

where $E$ is Young's modulus of rubber; $\sigma$ is the stress; $\varepsilon$ is the strain; $\bar{M}_{\mathrm{C}}$ is the average molecular weight between the crosslinked nodes; $\rho$ is the rubber elastomer density; $R_{\text {air }}$ is the gas constant; and $T$ is thermodynamic temperature. While considering the irrationality of the hypothesis and crosslinking defects, the derivation results are equivalent to multiplying by a correction term; however, it does not change the physical nature and basic conclusion derived above, and hence the correction term problem can be ignored here.

2.2. Calculation of Young's Modulus at Different Temperatures. Owing to the complexity of the composition and structure of rubber materials, it is difficult to measure $\bar{M}_{\mathrm{C}}$ directly. However, $\bar{M}_{\mathrm{C}}$ can be calculated from Young's modulus at a specific temperature and small deformation; this method can be used to derive the expression for $\bar{M}_{\mathrm{C}}$. The formula for $\bar{M}_{\mathrm{C}}$ can be used to further derive Young's modulus of rubber at different temperatures without measuring the microscopic parameters.

Using equation (1), the formula for Young's modulus can be obtained as follows:

$$
\begin{aligned}
\bar{M}_{c} & =\frac{3 \rho_{C} R T_{C}}{E_{C}}, \\
E & =\frac{3 \rho R T}{\bar{M}_{c}}=\frac{\rho T E_{C}}{\rho_{C} T_{C}},
\end{aligned}
$$

where $T_{C}$ is the room temperature, which is set to $25 \pm 2^{\circ} \mathrm{C}$; $\rho_{C}$ is the density at room temperature; and $E_{\mathrm{C}}$ is Young's modulus at room temperature.

The density of rubber varies with temperature, as the volume changes when the temperature changes. The linear thermal expansion coefficient of rubber is expressed as $\alpha$. Because $\alpha$ is extremely small, the volume expansion coefficient can be expressed as $(1+\alpha)^{3}-1 \approx 3 \alpha$. Thus, the following formula can be derived:

$$
\begin{aligned}
{\left[1+3 \alpha\left(T-T_{C}\right)\right] \rho } & =\rho_{C}, & & T>T_{C}, \\
\rho & =\left[1+3 \alpha\left(T_{C}-T\right)\right] \rho_{C}, & & T<T_{C} \\
\rho & =\rho_{C}, & & T=T_{C} .
\end{aligned}
$$

Furthermore, the following formula can be derived: 


$$
\begin{array}{ll}
\frac{\rho}{\rho_{C}}=\frac{1}{\left[1+3 \alpha\left(T-T_{C}\right)\right]}, & T>T_{C}, \\
\frac{\rho}{\rho_{C}}=\left[1+3 \alpha\left(T_{C}-T\right)\right], & T<T_{C}, \\
\frac{\rho}{\rho_{C}}=1, \quad T=T_{C} . &
\end{array}
$$

Setting $\Delta T=T-T_{\mathrm{C}}$, when $T<T_{\mathrm{C}}$, the following formula can be obtained:

$$
\begin{aligned}
1+3 \alpha\left(T_{C}-T\right) & =1-3 \alpha \Delta T=\frac{(1-3 \alpha \Delta T)(1+3 \alpha \Delta T)}{1+3 \alpha \Delta T} \\
& =\frac{1-9 \alpha^{2} \Delta T^{2}}{1+3 \alpha \Delta T} \approx \frac{1}{1+3 \alpha \Delta T} .
\end{aligned}
$$

According to equations (4) and (5), when $\alpha$ is extremely small and an approximation can be used, equation (5) can be simplified as follows:

$$
E=\frac{T E_{C}}{(1+3 \alpha \Delta T) T_{C}} .
$$

From equation (6), it can be seen that for a rubber spring with cross-linked rubber, when the temperature rises in a certain range, Young's modulus increases, which is consistent with the characteristics of a cross-linked polymer. In addition, owing to the nonlinearity of rubber, the stiffness will decrease gradually with increase in temperature when the pressure is relatively high, which is also consistent with the actual experimental results. The change in Young's modulus and stiffness do not always correspond to each other; this will be discussed in detail later.

2.3. Correction in Young's Modulus at Low Temperatures. Based on molecular physics theory for rubber materials, the following analysis is conducted. At high temperatures, the degree of cross-linking of rubber increases gradually with increase in temperature, which leads to an increase in hardness and Young's modulus of the rubber. If the temperature increases further, the molecular chain of the polymer will break. It is not necessary to consider the role of degradation, as the engineering application range is normally not higher than $60^{\circ} \mathrm{C}$. When the temperature of rubber is lower, the molecular activity is weakened, and the rubber will crystallize. This condition leads to an increase in Young's modulus of the rubber at low temperatures.

When a normal cross-linked rubber is at a temperature above $0^{\circ} \mathrm{C}$, the effect of crystallization is significantly weak. Young's modulus and stiffness of the rubber increase significantly with decrease in temperature when the temperature is below $0^{\circ} \mathrm{C}$. Therefore, $0^{\circ} \mathrm{C}$ is selected as the transition point in certain theoretical studies. In this study, it is considered that when the temperature is higher than $0^{\circ} \mathrm{C}$, Young's modulus changes as per equation (6), and when the temperature is lower than $0^{\circ} \mathrm{C}$, Young's modulus has a certain functional relationship with the ambient temperature.

Ding et al. [22] conducted correction analysis based on $20^{\circ} \mathrm{C}$. In the present study, correction analysis was conducted based on $0^{\circ} \mathrm{C}$. For considering temperature as the variable, the ratio of the secant Young's modulus of rubber at low temperature to that at $0^{\circ} \mathrm{C}$ was defined as the temperature coefficient $\varphi$, which can be expressed as follows:

$$
\begin{gathered}
\varphi=\frac{E}{E_{0}}, \\
\Delta T_{1}=T-T_{0},
\end{gathered}
$$

where $E$ is Young's modulus at $0^{\circ} \mathrm{C}$, and $T_{0}$ is the thermodynamic temperature at $0^{\circ} \mathrm{C}(273.15 \mathrm{~K})$. To simplify the calculation and obtain a certain fitting accuracy, the temperature coefficient is described by a polynomial function given as follows:

$$
\varphi=a_{0}+\sum_{i=1}^{n} a_{i}\left(-0.1 \Delta T_{1}\right)^{i},
$$

where $n$ is the order of the polynomial and $a_{i}$ is the polynomial coefficient. To avoid too big error while fitting the coefficient, the temperature difference is corrected. Combining (6)-(8), when $T<273.15 \mathrm{~K}, E$ is given by

$$
E=E_{0} \varphi=\frac{T_{0} E_{C}}{(1+75 \alpha) T_{C}} \varphi .
$$

Therefore,

$$
\begin{aligned}
& E=\frac{T E_{C}}{(1+3 \alpha \Delta T) T_{C}}, \quad T \geq 273.15 \mathrm{~K}, \\
& E=\frac{T_{0} E_{C}}{(1+75 \alpha) T_{C}} \varphi, \quad T<273.15 \mathrm{~K} .
\end{aligned}
$$

From the experimental data, by fitting a fourth-order temperature correction coefficient, the following equation can be obtained:

$$
\begin{aligned}
a_{0}= & 1.00197, \\
a_{1}= & 0.83289, \\
a_{2}= & -0.4072, \\
a_{3}= & 0.09596, \\
a_{4}= & -0.00735, \\
\varphi= & 1.00197-8.3289 \times 10^{-2} \Delta T_{1}-4.072 \\
& \times 10^{-3} \Delta T_{1}^{2}-1.3264 \times 10^{-4} \Delta T_{1}^{3}-7.35 \times 10^{-7} \Delta T_{1}^{4} .
\end{aligned}
$$

\section{Calculation of Circular Rubber Spring Stiffness}

3.1. Empirical Formula. In the development of rubber spring for railway vehicles, the stiffness of rubber spring is the most important parameter that designers and developers must 
consider. Based on vast experience, researchers have developed a simplified stiffness calculation method for rubber springs with a certain accuracy. In the bogies of railway vehicles, a circular rubber pad is commonly used as the rubber spring. Hence, in the following section, the circular rubber pad is taken as an example. For a circular rubber spring, when the deformation is extremely small, the empirical formula for static stiffness $K_{\mathrm{c}}$ is as follows:

$$
K_{c}=\frac{A_{c} \mu E}{H},
$$

where $A_{c}$ is the bearing area, $E$ is Young's modulus, $H$ is the height of the rubber pad, $\mu$ is the vertical shape coefficient, and $\mu=1+2 S^{2}$ (the approximate formulas given by different research institutes are slightly different). The area ratio $S$ can be calculated using the following formula:

$$
S=\frac{A_{c}}{A_{f}}=\frac{\pi\left(R^{2}-r^{2}\right)}{2 \pi(R+r) H}=\frac{R-r}{2 H},
$$

where $A_{\mathrm{f}}$ is the total free area that can be obtained by adding the inner and outer areas of the ring, $R$ is the outer radius of the rubber pad, and $r$ is the inner radius of the rubber pad. Further study shows that the empirical formula for the circular rubber spring is as follows:

$$
K_{c}=\frac{\pi r^{2} E\left(H^{2}+(1 / 2) r^{2}\right)}{H^{3}}=\frac{\pi r^{2} E}{H}+\frac{\pi r^{4} E}{2 H^{3}} .
$$

3.2. Calculation Based on the Hypothesis of Rectangular Deformation. During the compression of the circular rubber pad, the size of the rubber pad changes continuously. The stiffness $K_{c}$ is related to deformation and not a fixed value; thus, the static stiffness of the rubber spring is nonlinear. While calculating the free area, we assume that the rubber pad remains as a regular rectangular ring after the change. Assuming that the change in the inner radius is the same as that in the outer radius, we get $R_{1}-R=r-r_{1}=d . R_{1}$ is the outer radius of the rubber pad after compression, $r_{1}$ is the inner radius after compression, $R$ is the outer radius before compression, $r$ is the inner radius before compression, and $d$ is the change in radius. As the volume remains constant, we get

$$
\begin{aligned}
\pi\left(R_{1}^{2}-r_{1}^{2}\right)\left(H-h_{\text {pre }}\right) & =\pi\left(R^{2}-r^{2}\right) H, \\
d & =\frac{1}{2} \frac{(R-r) h_{\text {pre }}}{H-h_{\text {pre }}}, \\
R_{1} & =R+\frac{1}{2} \frac{(R-r) h_{\text {pre }}}{H-h_{\text {pre }}}, \\
r_{1} & =r-\frac{1}{2} \frac{(R-r) h_{\text {pre }}}{H-h_{\text {pre }}} .
\end{aligned}
$$

In the formula, $H$ is the initial height of the rubber pad and $h_{\text {pre }}$ is the precompression value. The formula is modified as follows:

$$
\begin{aligned}
S_{1}= & \frac{A_{c 1}}{A_{f 1}}=\frac{\pi\left(R_{1}^{2}-r_{1}^{2}\right)}{2 \pi\left(R_{1}+r_{1}\right)\left(H-h_{\mathrm{pre}}\right)}=\frac{R_{1}-r_{1}}{2\left(H-h_{\mathrm{pre}}\right)}, \\
K_{c 1}= & \frac{A_{c 1} \mu_{1} E}{H-h_{\mathrm{pre}}}=\frac{A_{c 1}\left(1+2 S_{1}^{2}\right) E}{H-h_{\mathrm{pre}}}=\frac{\pi\left(R_{1}^{2}-r_{1}^{2}\right) E}{H-h_{\mathrm{pre}}} \\
& \cdot\left[1+\frac{\left(R_{1}-r_{1}\right)^{2}}{2\left(H-h_{\mathrm{pre}}\right)^{2}}\right]
\end{aligned}
$$

where $S_{1}$ is the area ratio under the assumption of rectangular shape, $A_{f 1}$ is the free area, $K_{c 1}$ is the stiffness, $A_{c 1}$ is the bearing area, and $\mu_{1}$ is the vertical shape coefficient under the assumption of rectangular shape.

The static hyperelastic force $F_{c 1}$ is given by

$$
F_{c 1}=\int_{0}^{h_{\text {pre }}} K_{c 1} \mathrm{~d} z=\int_{0}^{h_{\text {pre }}} \frac{\pi\left(R_{1}^{2}-r_{1}^{2}\right) E}{H-z}\left[1+\frac{\left(R_{1}-r_{1}\right)^{2}}{2(H-z)^{2}}\right] \mathrm{d} z \text {. }
$$

Normally, owing to end friction, the change in the bearing area of the rubber pad after deformation is less than the calculated value; the bearing area of the rubber pad with rubber cover plate remains the same as the original value, but the change in the free area is larger. If the influence of rubber cover is considered, the bearing area should be the same as the original value, given by $A_{c 1}=A_{c}=\pi\left(R^{2}-r^{2}\right)$.

\subsection{Hypothesis of Elliptical Shape and Convexity Correction} Coefficient. The deformation of the circular rubber pad is irregular during compression, and hence the assumption of cylindrical deformation of the rubber pad is unrealistic; it should be close to elliptical deformation. Figure 1(a) shows the initial state of the circular rubber spring, Figure 1(b) shows the hypothetical state of rectangular deformation, and Figure 1(c) shows the hypothetical state of semiellipse for practical purposes.

During the deformation, the rubber cover is regarded as a rigid body, and hence the bearing area of the rubber pad does not change, but the free area of the rubber pad changes. When the rubber pad is deformed, the middle part protrudes outward; it is assumed that the bulging part of the rubber pad is a semiellipse in the vertical section, and that the semiaxes of the inner and outer semiellipses are equal, as shown in Figure 1(c). Taking the center of rubber pad as the origin of the rectangular coordinate system, let the semitransverse axis of the expanded semiellipse be represented by $a$ and the semivertical axis be represented by $b$. The outline of the vertical tangent of the rubber spring is shown in Figure 2.

The analytic geometry formulas are as follows: 


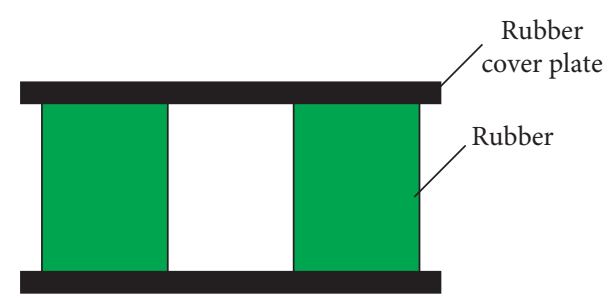

(a)

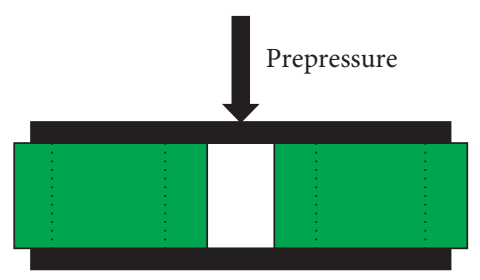

(b)

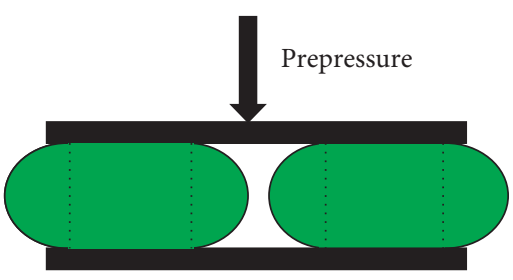

(c)

FIGURE 1: Diagram of circular rubber pad when compressed.

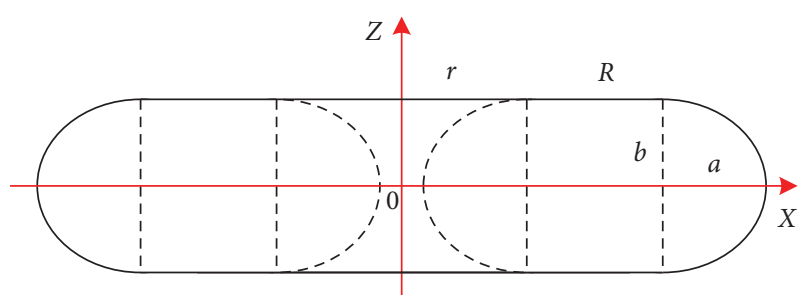

Figure 2: Diagram of geometric contour of circular rubber pad when elliptical shape is assumed.

$$
\begin{aligned}
& \frac{(x-R)^{2}}{a^{2}}+\frac{z^{2}}{b^{2}}=1, \quad R \leq x \leq R+a, \\
& z= \pm b, \quad-R<x<-r \cup r<x<r, \\
& \frac{(x-r)^{2}}{a^{2}}+\frac{z^{2}}{b^{2}}=1, \quad r-a \leq x \leq r, \\
& \frac{(x+r)^{2}}{a^{2}}+\frac{z^{2}}{b^{2}}=1, \quad-r \leq x \leq-r+a, \\
& \frac{(x+R)^{2}}{a^{2}}+\frac{z^{2}}{b^{2}}=1, \quad-R-a \leq x \leq-R .
\end{aligned}
$$

The volume enclosed by the outer elliptic curve is represented by $V_{\text {out }}$, and the volume enclosed by the inner elliptic curve is represented by $V_{\text {in }}$. Considering the right half, the following formulas can be derived:

$$
\begin{aligned}
V & =\pi\left(R^{2}-r^{2}\right) H=V_{\text {out }}-V_{\text {in }}=2 \int_{0}^{b} \pi\left(x_{1}^{2}-x_{2}^{2}\right) \mathrm{d} z \\
& =2 \pi \int_{0}^{b}\left[\left(R+\frac{a}{b} \sqrt{b^{2}-z^{2}}\right)^{2}-\left(r-\frac{a}{b} \sqrt{b^{2}-z^{2}}\right)^{2}\right] \mathrm{d} z \\
& =2 \pi\left(R^{2} b+\frac{2}{3} a^{2} b+\frac{1}{2} a b \pi R\right)-2 \pi\left(r^{2} b+\frac{2}{3} a^{2} b-\frac{1}{2} a b \pi r\right) \\
& =2 \pi b\left(R^{2}-r^{2}\right)+a b \pi(R+r) .
\end{aligned}
$$

Hence, $a$ can be solved as

$$
a=\frac{(R-r)(H-2 b)}{b},
$$

and $b$ is given by

$$
b=\frac{H-h_{\text {pre }}}{2} .
$$

Hence, $a$ is given by

$$
a=2 \frac{(R-r) h_{\text {pre }}}{H-h_{\text {pre }}} .
$$

The free area of the circular rubber pad, designated as $A_{f 2}$, can be calculated as follows:

$$
\begin{aligned}
A_{f 2} & =4 \pi \int_{0}^{b}\left(x_{1}+x_{2}\right) \mathrm{d} z=4 \pi \int_{0}^{b} x_{1} \mathrm{~d} z+4 \pi \int_{0}^{b} x_{2} \mathrm{~d} z \\
& =4 \pi \int_{0}^{b}\left(R+\frac{a}{b} \sqrt{b^{2}-z^{2}}\right) d z+4 \pi \int_{0}^{b}\left(r-\frac{a}{b} \sqrt{b^{2}-z^{2}}\right) \mathrm{d} z \\
& =4 b \pi R+a b \pi^{2}+\left(4 b \pi r-a b \pi^{2}\right)=4 b \pi(R+r) \\
& =2 \pi(R+r)\left(H-h_{\text {pre }}\right) .
\end{aligned}
$$

Comparing the free area in the case of ellipse hypothesis with that in the case of rectangle hypothesis, it can be observed that

$$
A_{f 2}=A_{f 1}=2 \pi(R+r)\left(H-h_{\text {pre }}\right) .
$$

Equation (24) is based on the assumption that the internal and external deformations are the same in both rectangular and elliptical deformation cases. When this assumption is not valid, then $A_{f 2} \neq A_{f 1}$; however, the difference between $A_{f 2}$ and $A_{f 1}$ is always extremely small. Notably, the calculation error in the case of rectangle hypothesis cannot be corrected only using the elliptical shape of the free area. Therefore, when the ellipse hypothesis is adopted, the formula can be further modified using the degree of elliptical deformation.

In practice, the stiffness of rubber increases with increase in stress. This is because the increase in stress leads to a change in both geometric and material nonlinearity. When the rubber element is compressed, the specimen becomes anisotropic, as the molecular chain is oriented more in the transverse direction.

The higher the degree of compression of the circular rubber pad, the greater the degree of protrusion of the rubber parts inward and outward, and the greater the change in the properties of the rubber. This change can be expressed 
by the convexity coefficient $\mu_{\text {con }}$, which can be defined as follows:

$$
\mu_{\mathrm{con}}=1+\frac{a}{b}
$$

The stiffness of the rubber pad, $K_{\mathrm{c} 2}$, is given by

$$
\begin{aligned}
K_{c 2} & =\frac{A_{c}\left(1+2 S_{2}^{2}\right) E}{H-h_{\text {pre }}} \mu_{2}=\frac{\pi\left(R^{2}-r^{2}\right) E}{H-h_{\text {pre }}}\left[1+\frac{\left(R^{2}-r^{2}\right)^{2}}{8 b^{2}(R+r)^{2}}\right]\left(1+\frac{a}{b}\right) \\
& =\frac{\pi\left(R^{2}-r^{2}\right) E}{H-h_{\text {pre }}}\left[1+\frac{(R-r)^{2}}{2\left(H-h_{\text {pre }}\right)^{2}}\right]\left(1+\frac{a}{b}\right) .
\end{aligned}
$$

The prepressure $F_{c 2}$ is given by

$$
\begin{aligned}
F_{c 2}= & \int_{0}^{h_{\text {pre }}} K_{c} \mathrm{~d} z=\int_{0}^{h_{\text {pre }}} \frac{\pi\left(R^{2}-r^{2}\right) E}{H-z}\left[1+\frac{(R-r)^{2}}{2(H-z)^{2}}\right] \\
& \cdot\left(1+\frac{a}{b}\right) \mathrm{d} z .
\end{aligned}
$$

In the above formulas, $b$ and $a$ correspond to the definitions in formulas (21) and (22), respectively, and $h_{\text {pre }}$ in $a$ and $b$ is replaced by the variable $z$.

\section{Variation in Geometric Nonlinearity of Rubber Spring with Temperature}

During the operation of railway vehicle bogies, the temperature of rubber components changes continuously, normally in the range of $-60^{\circ} \mathrm{C}$ to $+60^{\circ} \mathrm{C}$. If the temperature changes, the volume of rubber will change because of thermal expansion and contraction.

Considering the room temperature $T_{\mathrm{C}}$ as the standard condition, when the temperature change is $\Delta T$, the volume of the circular rubber spring expands to $(1+\alpha \Delta T)^{3} \pi\left(R^{2}-r^{2}\right) H$, and the theoretical height changes to $(1+\alpha \Delta T) H$. However, owing to the restriction imposed by the rubber cover, it is considered that the bearing surface of the rubber remains unchanged (the thermal expansion of steel is much lower than that of rubber), and the lateral changes are limited to a certain extent. If the temperature rises, the rubber will protrude outwards; otherwise it will become concave inwards. Equation (19) can be modified as follows:

$$
\begin{aligned}
(1+\alpha \Delta T)^{3} V & =(1+\alpha \Delta T)^{3} \pi\left(R^{2}-r^{2}\right) H=V_{\text {out }}-V_{\text {in }} \\
& =2 \int_{0}^{b} \pi\left(x_{1}^{2}-x_{2}^{2}\right) \mathrm{d} z \\
& =2 \pi b\left(R^{2}-r^{2}\right)+a b \pi(R+r) .
\end{aligned}
$$

From equation (28), it can be concluded that

$$
a=\frac{(R-r)\left[(1+\alpha \Delta T)^{3} H-2 b\right]}{b},
$$

where

$$
b=\frac{(1+\alpha \Delta T) H-h_{\text {pre }}}{2} .
$$

The free area $A_{f 2}$ is given by

$$
A_{f 2}=4 b \pi(R+r)=2 \pi(R+r)\left[(1+\alpha \Delta T) H-h_{\text {pre }}\right] \text {. }
$$

The shape coefficient $\mu_{2}$ is given by

$$
\mu_{2}=1+2 S_{2}^{2}=1+2\left(\frac{A_{c}}{A_{f 2}}\right)^{2}=1+\frac{(R-r)^{2}}{2\left[(1+\alpha \Delta T) H-h_{\text {pre }}\right]^{2}} \text {. }
$$

When the temperature changes, equations (26) and (27) can be further modified as follows:

$$
\begin{aligned}
& K_{c 2}=\frac{A_{c}\left(1+2 S_{2}^{2}\right) E}{(1+\alpha \Delta T) H-h_{\text {pre }}} \mu_{2}=\frac{\pi\left(R^{2}-r^{2}\right) E}{(1+\alpha \Delta T) H-h_{\text {pre }}}\left[1+\frac{(R-r)^{2}}{\left.2\left[(1+\alpha \Delta T) H-h_{\text {pre }}\right]^{2}\right]\left(1+\frac{a}{b}\right),}\right. \\
& F_{c 2}=\int_{0}^{h_{\text {pre }}} K_{c 2} \mathrm{~d} z=\int_{0}^{h_{\text {pre }}} \frac{\pi\left(R^{2}-r^{2}\right) E}{(1+\alpha \Delta T) H-z}\left[1+\frac{(R-r)^{2}}{2[(1+\alpha \Delta T) H-z]^{2}}\right]\left(1+\frac{a}{b}\right) \mathrm{d} z .
\end{aligned}
$$

Young's modulus $E$ in equation (33) is calculated using equation (10), and the parameters $a$ and $b$ are calculated using equations (29) and (30). The variable $h_{\text {pre }}$ in $a$ and $b$ is replaced by the variable $z$.

Equation (33) shows that when the pressures are given instead of the preloading amplitude, the preloading amplitude can be calculated by the inverse function $h_{\text {pre }}=f^{-1}\left(F_{c 2}\right)$; this function can be derived from $F_{c 2}=f\left(h_{\text {pre }}\right)$. The nonlinear stiffness can be calculated using the preloading amplitude $h_{\text {pre }}$. As it is difficult to calculate the inverse function, it is normally obtained by numerical calculation using a computer.

\section{Comparison of Results of Test and Analysis}

The rubber pad of a Chinese standard EMU bogie is taken as an example in the present study. As shown in Figure 3, the rubber spring has a combined metal cover at both ends. Vertical static compression tests were conducted at different temperatures and pressures, and actual data were obtained. 


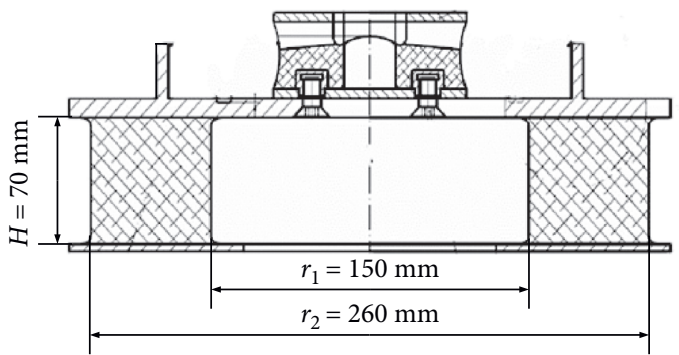

FIgURE 3: Rubber pad of a Chinese standard EMU bogie.

When the hardness of the rubber spring is $55 \mathrm{HS}$, according to the empirical formula for the hardness and Young's modulus in the mechanical design manual [28], Young's modulus $E_{\mathrm{C}}$ is $2.28 \mathrm{MPa}$ at room temperature without preloading. For this circular rubber pad, the coefficient of thermal expansion is $\alpha=6.6 \times 10^{-4} \mathrm{~K}^{-1}$. Using equation (10), Young's modulus $E$ can be calculated at different temperatures, as shown in Figure 4.

In Figure 4, when the temperature is above $0^{\circ} \mathrm{C}$, Young's modulus increases slowly with increase in temperature, which conforms to the theoretical behavior of cross-linked rubber. When the temperature is below $0^{\circ} \mathrm{C}$, Young's modulus increases rapidly with decrease in temperature. When the temperature is near $0^{\circ} \mathrm{C}$, there may be large errors and insufficient smoothness in the curve owing to piecewise design and ideal hypothesis. Although there are some defects, the basic trend of this curve reflects the theoretical analysis and test results. To verify the correctness of equation (33), the differences between theoretical model results and measured data are compared and analyzed. These results are shown in Figure 5. Figure 5(a) shows the curve at different pressures at a temperature of $25^{\circ} \mathrm{C}$, and Figure 5(b) shows the curve at different temperatures at a pressure of $57 \mathrm{kN}$.

This section covers the thermostatic analysis. Figure 5(a) shows that the difference between the original model and the convexity coefficient correction model is more significant with increase in prepressure. The modified formula for the additional convexity coefficient can be a good approximation at normal pressures in the range of $45-85 \mathrm{kN}$ (based on the actual working conditions of trains); the maximum error is $-10.86 \%$ and the root mean square error is $7.71 \%$. The error in the original shape coefficient model is between $-39.2 \%$ and $-48.18 \%$ in the range of pressures normally used, and the error increases with increase in pressure, but the accuracy is higher when the prepressure is low. Although there is a root mean square error of $7.71 \%$ in the calculation using the modified formula for the additional convexity coefficient, all the coefficients are calculated using their original physical quantities and geometric sizes. From the above analysis, it can be noted that the convexity coefficient correction formula is effective and accurate in reflecting the stiffness change caused by the change in pressure in the normal range of pressures. When the prepressure increases further, the error also tends to increase, but this is not within the commonly used prepressure range in engineering.

This section covers the constant prepressure analysis. Figure 5(b) shows that the correction formula for convexity

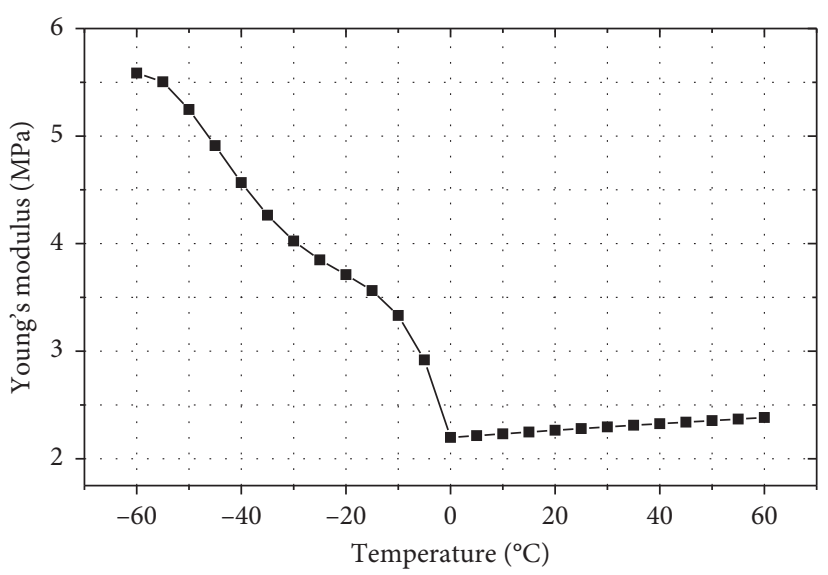

FIgUre 4: The relationship between Young's modulus and temperature of rubber spring.

coefficient is more accurate in the range of $-60^{\circ} \mathrm{C}$ to $+60^{\circ} \mathrm{C}$. When the temperature is above $0^{\circ} \mathrm{C}$ and the prepressure is in the normal range, the calculation result obtained using the modified formula is close to that obtained using the original empirical formula. The maximum error with the modified formula is $11.30 \%$, and the root mean square error is $8.93 \%$. Although the error increases gradually when the temperature is higher than $60^{\circ} \mathrm{C}$, it is generally not considered. When the temperature is below $0^{\circ} \mathrm{C}$, the maximum error with the revised formula is $6.75 \%$, and the root mean square error is $4.64 \%$; the maximum error with the original empirical formula is $18.84 \%$, and the root mean square error is $11.06 \%$. From the above analysis, it can be observed that (33) can reflect the change in stiffness with temperature.

The theoretical data as per the modified formula and experimental data are shown in Figures 6 and 7. Notably, the modified equation (33) has a large error of approximately $20.2 \%$ at the lowest temperature and the highest preload. However, the error is less than $10 \%$ under the normal range of temperature and preload.

Based on the above analysis, it can be inferred that equation (33) can better reflect the change in actual stiffness with temperature as well as prepressure. Therefore, equation (33) is reasonable and effective for describing the change in static stiffness of the rubber pad under the influence of temperature and prepressure.

Using equation (33), the theoretical value of stiffness under a certain prepressure (or precompression amplitude) and at a certain temperature can be calculated, as shown in Figure 8 .

As shown in Figure 8, the stiffness of the rubber spring changes when the amplitude of pressure or compression changes; this is caused by the nonlinearity of stiffness. Notably, the stiffness decreases slightly with increase in temperature, when the temperature is above $0^{\circ} \mathrm{C}$ and the preload is below $45 \mathrm{kN}$; the stiffness increases slightly with increase in temperature, when the temperature is above $0^{\circ} \mathrm{C}$ and the preload is above $45 \mathrm{kN}$. This is mainly because temperature affects not only Young's modulus, but also the geometric nonlinearity of the rubber spring. When the temperature is lower than $0^{\circ} \mathrm{C}$, variations in pressure have 


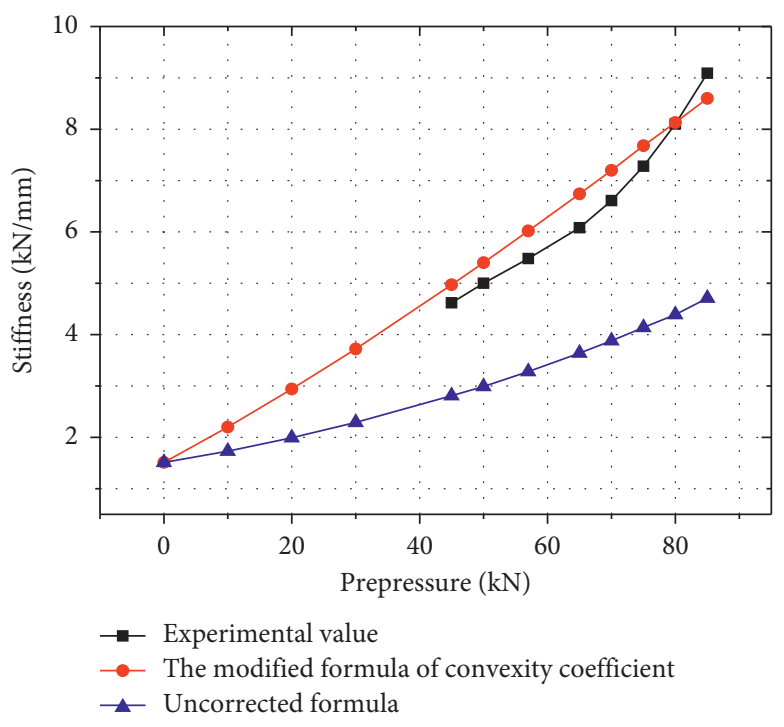

(a)

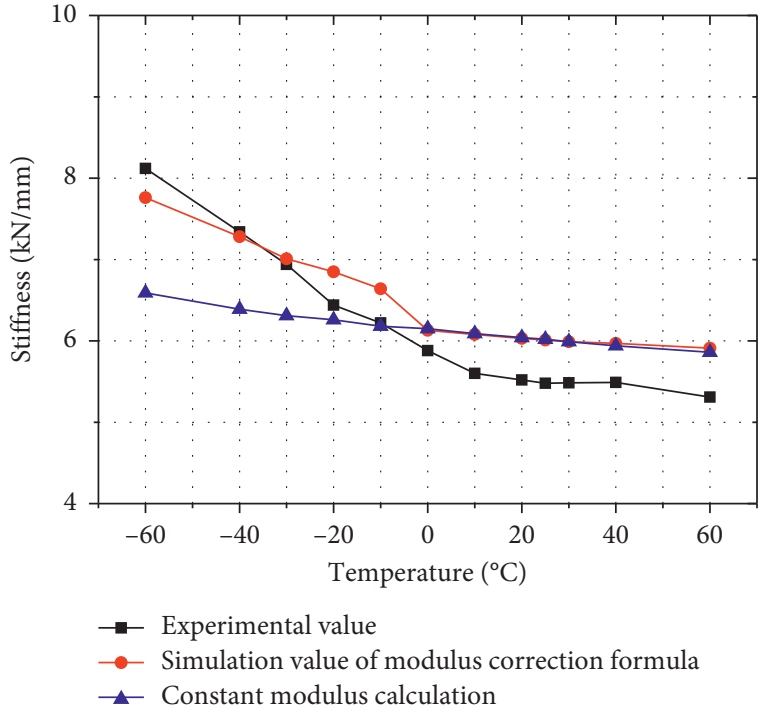

(b)

FiguRE 5: Comparison of measured values and theoretical model calculation. (a) Comparison of stiffness at different prepressures. (b) Comparison of stiffness at different temperatures.

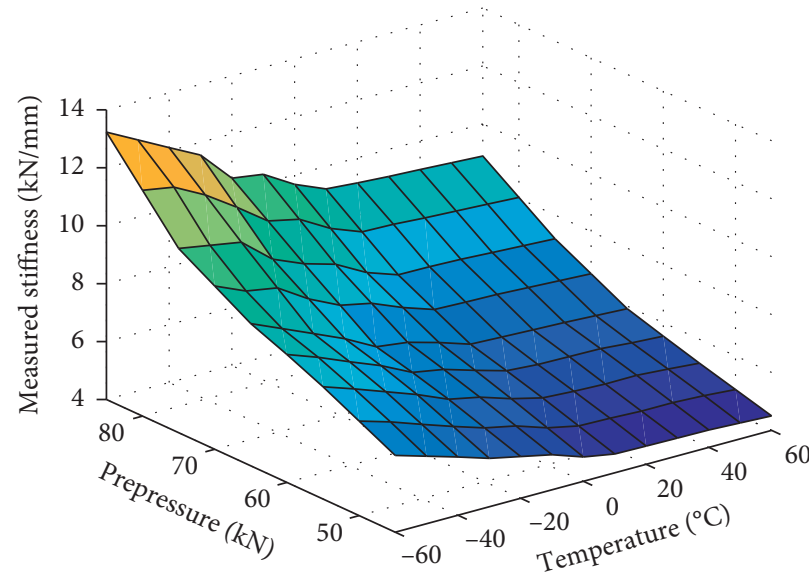

Figure 6: Surface diagram of measured stiffness.

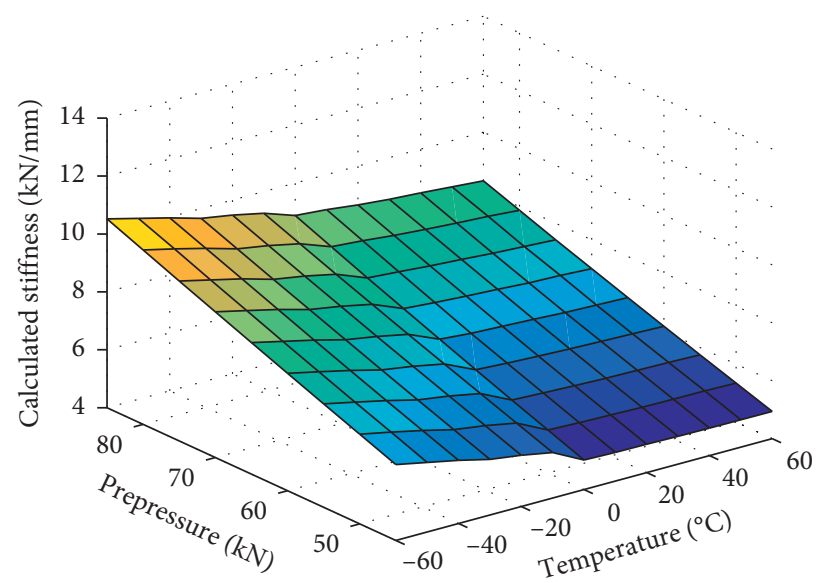

Figure 7: Surface diagram of calculated stiffness. 


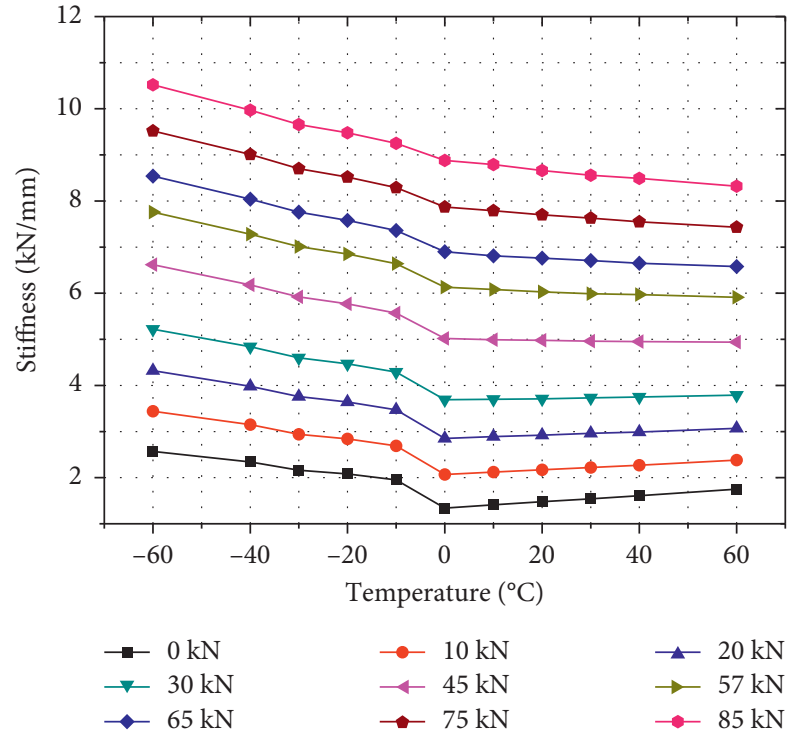

(a)

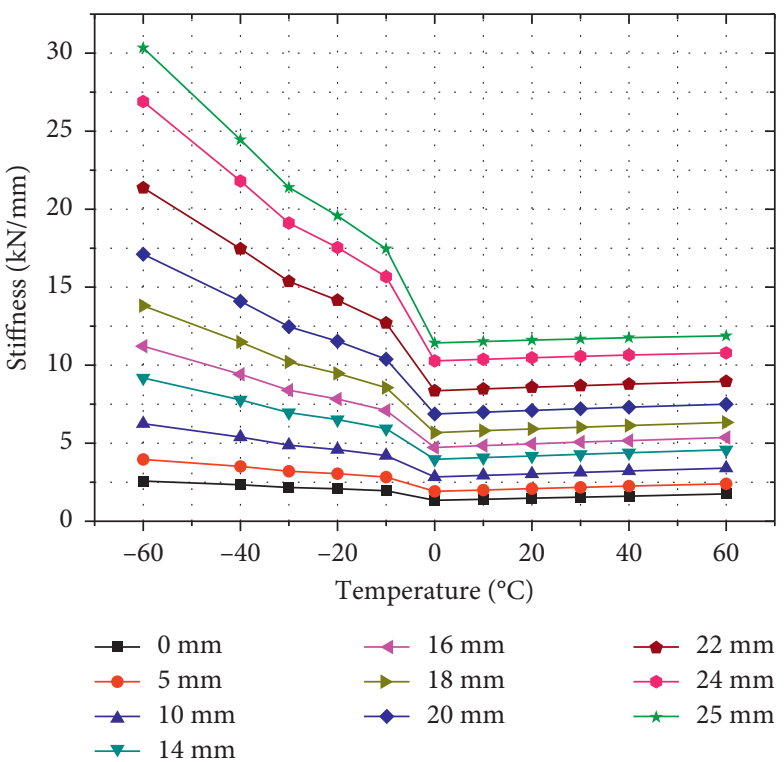

(b)

Figure 8: Curves for change in stiffness. (a) Stiffness variation under different temperatures and prepressures. (b) Stiffness variation under different temperatures and compression amplitudes.

little effect on the change trend of stiffness. In Figure 8(b), the stiffness hardly varies with temperature at the same precompression amplitude when the temperature is above $0^{\circ} \mathrm{C}$. When the temperature is lower than $0^{\circ} \mathrm{C}$, the greater the precompression amplitude, the more drastic is the change in stiffness with temperature.

\section{Conclusions}

In this thesis, the relationship between Young's modulus and temperature of rubber was studied, using the conformation statistical theory of polymer chains. At low temperature, a more effective Young's modulus correction formula is proposed. The modified Young's modulus is applied to the stiffness calculation formula for the rubber pad based on elliptical convexity coefficient correction and dimension correction. Finally, based on the test data of the circular rubber pad of a Chinese standard EMU bogie, a comparative analysis and theoretical research were conducted. The following conclusions can be drawn.

(1) There is a close relationship between Young's modulus and temperature in cross-linked rubber materials. When the temperature is above $0^{\circ} \mathrm{C}$, Young's modulus increases gradually with increase in temperature. When the temperature is below $0^{\circ} \mathrm{C}$, Young's modulus increases significantly with decrease in temperature owing to the influence of partial crystallization. Therefore, it is effective and reasonable to adopt variable Young's modulus in the calculations.

(2) In addition to the temperature factor at low temperature, the other parameters are derived from theory or calculated based on geometric parameters.
Thus, the convexity coefficient correction formula has good application value. The formula can also be applied to rubber pads of other sizes.

(3) The modified formula considers the influence of temperature and prepressure at the same time. The calculated values are close to the actual values, and the calculation error under normal working conditions is less than $10 \%$. Therefore, it can be considered that the convexity coefficient correction method is effective for stiffness calculation. Consideration of the effect of temperature has significantly improved the accuracy of the original formula.

(4) Different prepressures have the same effect on the change trend of low-temperature stiffness, and the stiffness increases significantly with decrease in temperature. However, the stiffness is less affected by temperature when the temperature is above $0^{\circ} \mathrm{C}$, but it increases slightly with increase in temperature when the preloading is small and decreases slightly with increase in temperature when the preloading is large. In theory, Young's modulus increases with temperature, but in most cases, the measured stiffness decreases with temperature. This seemingly contradictory phenomenon is explained by (33).

(5) There is a nonlinear relationship between prepressure and precompression amplitude. Their influence on stiffness is different.

The theory of static stiffness of rubber pad is of great significance in the study of dynamic performance of rubber and provides a method to further derive the formula for dynamic stiffness. The dynamic properties of rubber under the influence of temperature and prepressure will be paid more attention in the future research. 


\section{Data Availability}

The data used to support the findings of this study are available from the corresponding author upon request.

\section{Conflicts of Interest}

The authors declare that they have no conflicts of interest.

\section{Acknowledgments}

The authors would like to thank Editage (http://www.editage. cn) for English language editing. This research has been supported by the Youth Program of National Natural Science Foundation of China (Grant no. 51805450) and by the National Key R\&D Program of China (Grant nos. 2016YFB120404, 2018YFB1201700, and 2018YFE0201401-01)

\section{References}

[1] R. Luo, H. Shi, J. Guo, L. Huang, and J. Wang, "A nonlinear rubber spring model for the dynamics simulation of a high speed train," Vehicle System Dynamics, 2019.

[2] D. Zhang and S. Zhu, "A fractional derivative model for rubber spring of primary suspension in railway vehicle dynamics," ASCE-ASME Journal of Risk and Uncertainty in Engineering Systems, Part B: Mechanical Engineering, vol. 3, 2017.

[3] D.-w. Sun, Z.-g. Chen, G.-y. Zhang, and P. Eberhard, "Modeling and parameter identification of amplitude- and frequency-dependent rubber isolator," Journal of Central South University, vol. 18, no. 3, pp. 672-678, 2011.

[4] J. Wu and S. Wen-Bin, "Modeling and applications of dynamic characteristics for rubber isolators using viscoelastic fractional derivative model," Engineering Mechanics, vol. 25, no. 1, pp. 161-166, 2008, in Chinese.

[5] H. Shi and P. Wu, "A nonlinear rubber spring model containing fractional derivatives for use in railroad vehicle dynamic analysis," Proceedings of the Institution of Mechanical Engineers, Part F: Journal of Rail and Rapid Transit, vol. 230, no. 7, pp. 1745-1759, 2016.

[6] S. Mattias, "Rubber isolators-measurements and modeling using fractional derivatives and friction," in Proceedings of the SAE Technical Paper Series, Troy, MI, USA, December 2000.

[7] S. Mattias and K. Leif, "Non-linear behavior of a rubber isolator system using fractional derivatives," Vehicle System Dynamics, vol. 37, no. 3, pp. 217-236, 2002.

[8] M. Sjöberg and L. Kari, "Nonlinear isolator dynamics at finite deformations: an effective hyperelastic, fractional derivative, generalized friction model"' Nonlinear Dynamics, vol. 33, no. 3, pp. 323-336, 2003.

[9] L. Chen, G.-T. Yang, and Z.-Z. Huang, "Constitutive equations of incompressible nonlinear super-elastic material," Transaction of Beijing Institute of Technology, vol. 31, no. 1, pp. 30-34, 2011, in Chinese.

[10] Y. Q. Li and X.-L. Gao, "Constitutive equations for hyperelastic materials based on the upper triangular decomposition of the deformation gradient," Mathematics and Mechanics of Solids, vol. 24, no. 6, pp. 1785-1799, 2019.

[11] M. Gajewski, R. Szczerba, and S. Jemioło, "Modelling of elastomeric bearings with application of Yeoh hyperelastic material model," Procedia Engineering, vol. 111, pp. 220-227, 2015.
[12] M. Gajewski, "Estimation of the energy dissipation capability for chosen elastomers with application of DMA," Polymer Testing, vol. 68, pp. 405-414, 2018.

[13] D. Cardone and G. Gesualdi, "Experimental evaluation of the mechanical behavior of elastomeric materials for seismic applications at different air temperatures," International Journal of Mechanical Sciences, vol. 64, no. 1, pp. 127-143, 2012.

[14] A. Stevenson, "The influence of low-temperature crystallization on the tensile elastic modulus of natural rubber," Journal of Polymer Science: Polymer Physics Edition, vol. 21, no. 4, pp. 553-572, 1983.

[15] A. Stevenson, "Effect of elastic strain on the low temperature crystallization of rubber," Plastics \& Rubber Institute, vol. 9, 1987.

[16] P. Spanos, "Cure system effect on low temperature dynamic shear modulus of natural rubber," Rubber World, vol. 229, no. 2, pp. 22-27, 2003.

[17] H.-Q. Yu and X.-H. Liu, "Study on shear properties of lowtemperature modified nature rubber," Journal of Solid Rocket Technology, vol. 29, no. 3, pp. 222-224, 2006, in Chinese.

[18] T. L. Yu, D. Y. Yuan, and X. L. Sun, "The finished product of rubber bearing mechanical performance analysis in the low temperature," Applied Mechanics and Materials, vol. 178-181, pp. 2254-2259, 2012.

[19] W. Teng, F. Tan, and H. Shi, "Experimental study of high/low temperature effects on the dynamic performance of rubber spring for railway vehicles," Journal of Physics: Conference Series, vol. 1213, no. 5, 2019.

[20] R. Österlöf, L. Kari, and H. Wentzel, “Temperature dependency of a viscoplastic constitutive model for rubber with reinforcing fillers," Constitutive Models for Rubber IX, pp. 149-156, Boca Raton, FL, USA, 2015.

[21] Y. Lü, L. Guo, Z. Deng, and Y. Wang, “Temperature-dependent tensile behavior of silicon rubber using automated grid method," Polymeric Materials Science and Engineering, vol. 32, no. 2, pp. 104-108, 2016, in Chinese.

[22] Z. Ding, L. Mu, J. Bu, Y. Huang, and J. Zeng, "Stiffness prediction of rubber springs at lower temperature," Journal of Vibration and Shock, vol. 36, no. 14, pp. 66-70, 2017, in Chinese.

[23] L. Kari, "The non-linear temperature dependent stiffness of precompressed rubber cylinders," KGK-Kautschuk und Gummi Kunststoffe, vol. 55, no. 3, pp. 76-81, 2002.

[24] L. Kari, "An analytical temperature-dependent collocation model for preloaded rubber cylinders," The Journal of Strain Analysis for Engineering Design, vol. 37, no. 4, pp. 289-299, 2002.

[25] S.-Q. You, B. Liu, and Y.-L. Lou, "Low-temperature effect on deformation behavior of laminated rubber isolators," Journal of Northeastern University, vol. 26, no. 3, pp. 297-299, 2005, in Chinese.

[26] X.-Y. Sun, W.-G. Yang, M. Wang et al., "Compression stiffness ratio of rubber bearings under shear deformation," Engineering Mechanics, vol. 34, no. 1, pp. 58-68, 2017, in Chinese.

[27] Z. Y. Cheng, Z. D. Hu, and L. Li, "Study on the performance of lead rubber bearing considering vertical force correlation," International Journal of Multiphysics, vol. 12, no. 3, pp. 267-282, 2018.

[28] D. Cheng, Mechanical Design Manual, Chemical Industry Press, Beijing, China, 2008, in Chinese, Fifth edition. 